\title{
Guest Editorial: FAIR 2019 special issue
}

\author{
Deshendran Moodley $^{\mathrm{a}, \mathrm{b}}$ (iD) deshen@cs.uct.ac.za, Marelie H. Davel ${ }^{\mathrm{c}, \mathrm{b}}$ (D), \\ marelie.davel@nwu.ac.za \\ ${ }^{a}$ Department of Computer Science, University of Cape Town, South Africa \\ ${ }^{c}$ Faculty of Engineering, North-West University, South Africa \\ ${ }^{b}$ Centre for Artificial Intelligence Research, South Africa
}

In this special issue, we feature selected papers from the inaugural Forum for Artificial Intelligence Research (FAIR), established and hosted by the Centre for Artificial Intelligence Research (CAIR) ${ }^{1}$. FAIR 2019 was held at the UCT Graduate School of Business Conference Centre in Cape Town, between 3 and 6 December 2019.

The Department of Science and Technology's (DST) latest White Paper on Science, Technology and Innovation (2019) identifies Artificial Intelligence (AI) and advanced Information and Communication Technologies (ICTs) as priority areas for South Africa. It recognises that these technologies will change the way the South African society and economy function. The potential of AI is already being unlocked in key areas of South African society. For example, South Africa's power utility, Eskom, has identified AI as a future area for research and innovation, and is exploring the use of machine learning for real-time monitoring and fault prediction at their power stations (Bhugwandin et al., 2019). The South African Revenue Service is aggressively building an in-house AI capability for analysing and detecting non-compliance in tax returns (South African Revenue Services, 2020).

The South African AI research community has also grown substantially over the last few years. While AI is generally considered to be a subdiscipline of Computer Science (Stone et al., 2016), it is at heart multidisciplinary: active AI research groups in South African universities can be found in Computer Science, Engineering, Philosophy, Information Systems, Statistics and Applied Mathematics departments.

Within this context, FAIR was established to provide a venue for South African AI researchers from a broad range of disciplines to meet, interact and publish their work. Research contributions were solicited in five tracks, namely applications of AI, ethics and AI, knowledge representation, machine learning, and other topics in AI. A total of 72 submissions were received, consisting of full papers, work in progress and extended abstracts (of work under review or published elsewhere). Full paper submissions were blind reviewed by at least two independent reviewers from the relevant disciplines and 20 full papers were accepted for publication in the conference proceedings (Davel \& Barnard, 2019).

Moodley, D. and Davel, M.H. (2020). Guest Editorial: FAIR 2019 special issue [Guest Editorial]. South African Computer Journal 32(2), x-xiii. https://doi.org/10.18489/sacj.v32i2.915

Copyright (C) the author(s); published under a Creative Commons NonCommercial 4.0 License (CC BY-NC 4.0). $S A C J$ is a publication of the South African Institute of Computer Scientists and Information Technologists. ISSN 1015-7999 (print) ISSN 2313-7835 (online).

${ }^{1}$ http://cair.org.za 
Following the conference, a subset of the presented papers was selected (all papers selected by at least one reviewer for this purpose), and authors were invited to submit an extended version of their conference paper for this special issue. These papers underwent another round of reviews using the $S A C J$ guidelines, requiring at least a $30 \%$ extension, and aligned feedback from at least three reviewers. Nine papers were deemed to meet these requirements and appear in this special issue. The selected papers address a range of topics, from applications of AI, to more theoretical work in machine learning and knowledge representation and reasoning.

The first three papers describe applications of AI, specifically relevant in the South African context:

- In "Clustering residential electricity consumption data to create archetypes that capture household behaviour in South Africa" Wiebke Toussaint and Deshendran Moodley present a new approach for eliciting and capturing expert domain knowledge and application requirements to guide the clustering process. They illustrate the use of this technique to identify dominant patterns of electricity consumption behaviour in the DELMH dataset, the largest and most comprehensive database of household electricity consumption in South Africa.

- In "Pairwise networks for feature ranking of a geomagnetic storm model" Jacques Beukes and co-authors present a new neural network architecture that is intrinsically more interpretable than a standard fully-connected, feedforward network. They use the new architecture to model the expected geomagnetic disturbance based on solar wind measurements, and demonstrate that the feature rankings produced by this model agree with current understanding of the physics at play. This bodes well for future application to tasks that are not as well understood.

- In "Improved semi-supervised learning technique for automatic detection of South African abusive language on Twitter" Oluwafemi Oriola and Eduan Kotzé address the task of identifying implicitly or explicitly abusive language in the South African context. A semi-supervised approach is used, and the effect of different features and classifiers compared. Good performance is achieved on a novel dataset.

The next three papers address theoretical aspects of machine learning:

- In "Benign interpolation of noise in deep learning" Marthinus Theunissen and co-authors study the ability of deep neural networks to generalise well in the presence of noise, and show that this can be explained by the modular way in which samples are processed during training. They discuss how this perspective could be applicable to other open questions related to the generalisation ability of these models.

- In “Using Summary Layers to Probe Neural Network Behaviour" Marelie Davel explores the theoretical and empirical ability of a feedforward neural network to achieve perfect classification in the presence of a 2-node summary layer, and what insight this can provide with regard to the training process and generalisation properties of such networks. 
- In "Ht-index for empirical evaluation of the sampled graph-based Discrete Pulse Transform", Mark de Lancey and Inger Fabris-Rotelli describe improvements to the running time and memory requirements of the Roadmaker's Pavage algorithm. This algorithm can be used to obtain an approximation of the Discrete Pulse Transform, which, in turn, is useful for extracting features and textures in computer vision.

The final three papers address theoretical and practical aspects of Knowledge Representation \& Reasoning. All three papers deal with issues around defeasible reasoning, i.e. the handling of exceptions in logic-based systems.

- In "Algorithmic Definitions for KLM-Style Defeasible Disjunctive Datalog" Matthew Morris and co-authors analyse algorithms for defeasible reasoning in Datalog. They introduce Relevant Closure and Lexicographic Closure as alternatives for computing defeasible entailment and adapt both of the algorithms for Datalog. They found that Lexicographic Closure is still LM-rational, but that Relevant Closure does not satisfy some of the KLM properties.

- In "Defeasibility applied to Forrester's paradox" Julian Chingoma and Thomas Meyer examine the effectiveness of KLM-style defeasible reasoning and Propositional Typicality Logic in dealing with Deontic paradoxes in the legal domain.

- In “DDLV: A System for rational preferential reasoning for datalog" Michael Harrison and Thomas Meyer present an extension to Datalog, the DDLV system, for representing and reasoning with defeasible information. The system is shown to satisfy the KLM requirements for a rational consequence and can be used to efficiently reason about contradictory information in Datalog.

We would like to thank Philip Machanick as editor of SACJ for the assistance provided in compiling this special issue, the reviewers for the extensive feedback provided, and the authors for sharing their ideas on this platform.

\section{References}

Bhugwandin, K., Moodley, P. \& Naidoo, S. (2019). Eskom Research, Testing and Development Research Direction Report: a working document (RaDaR) 2019 - 2024 (tech. rep.). Eskom Holdings SOC. https:// www.eskom.co.za/OurCompany/SustainableDevelopment/ Pages/ResearchDirection.aspx

Davel, M. \& Barnard, E. (Eds.). (2019). Proceedings of the South African Forum for Artificial Intelligence Research (FAIR) (Vol. 2540). http://ceur-ws.org/Vol-2540/

Department of Science and Technology (DST). (2019). White paper on Science, Technology and Innovation (tech. rep.). Department of Science and Technology. https://www.dst.gov. za/images/2019/White_paper_web_copyv1.pdf 
South African Revenue Services. (2020). SARS takes a big step towards building a smart modern revenue authority [Press Release]. https://www.sars.gov.za/Media/MediaReleases/ Pages/1-February-2020-Smart-modern-revenue-authority.aspx

Stone, P., Brooks, R., Brynjolfsson, E., Calo, R., Etzioni, O., Hager, G., Hirschberg, J., Kalyanakrishnan, S., Kamar, E., Kraus, S., Leyton-Brown, K., Parkes, D., Press, W., Saxenian, A., Shah, J., Tambe, M. \& Teller, A. (2016). Artificial Intelligence and life in 2030: One hundred year study on Artificial Intelligence (tech. rep.). Stanford University. http://ai100. stanford.edu/2016-report 